\title{
Motion of Solid Grains During Magnetic Field- Assisted Directional Solidification
}

\author{
JIANG WANG, XIN LIN, YVES FAUTRELLE, HENRI NGUYEN-THI, \\ and ZHONGMING REN
}

In this paper, we report the visible evidence for thermoelectric magnetic forces (TEMFs) during magnetic field-assisted directional solidification, and their potential to control the motion of solid grains(dendrite fragments or equiaxed grains). These motions are observed by means of synchrotron X-ray radiography and compared with analytic calculations for a spherical particle's motion driven only by TEMFs, which confirms that the observed solid grain motions are the combined result of the TEMFs and gravity. We also carried out corresponding 3D numerical simulations to validate the calculations and further prove our conclusion that TEMF acts on the solid grain and affects its motion trajectory.

https://doi.org/10.1007/s11663-018-1199-6

(c) The Minerals, Metals \& Materials Society and ASM International 2018

\section{INTRODUCTION}

MAGNETIC field can influence the flow of a conducting liquid without direct contact in accordance with the theory of magnetohydrodynamics, therefore applying magnetic fields to control flows in the melt during solidification of metals has gained increasing attention over the last half a century. ${ }^{[1]}$ Although extensive experimental and theoretical studies ${ }^{[2,3]}$ have yielded widely accepted conclusions that applying static magnetic fields to solidification processes can shift the liquid-to-solid phase-transformation temperature, ${ }^{[4]}$ align crystal/grain orientation, ${ }^{[5]}$ redistribute the solute, ${ }^{[6]}$ primary phase or inclusion ${ }^{[7]}$ and damp flows in the melt, ${ }^{[8,9]}$ our understanding of magnetic field-assisted solidification is still far from complete. For instance, contrary to damping the melt flows, Shercliff $^{[10]}$ pointed out that applying a static magnetic field can also induce flows in the melt by interacting with thermoelectric currents at the vicinity of the solid-liquid interface during solidification. Such flows, named thermoelectric magnetohydrodynamic flows (TEMHDFs), have been extensively studied in the context of pumping

JIANG WANG and ZHONGMING REN are with the State Key Laboratory of Advanced Special Steel, Shanghai University, 200072 Shanghai, P.R. China. Contact e-mail: jiangwang@i.shu.edu.cn YVES FAUTRELLE is with the SIMAP/EPM, 1130 rue de la Piscine BP 75 ENSEEG, 38402 St-Martin d Heres, France. HENRI NGUYEN-THI is with the Aix Marseille University, Campus Saint-Jerome, Case 142, 13397 Marseille Cedex 20, France. XIN LIN is with the State Key Laboratory of Solidification Processing, Northwestern Polytechnical University, Xi'an 710072, P.R. China.

Manuscript submitted August 22, 2017. or stirring liquid metal coolants in nuclear reactors. ${ }^{[1]}$ Research on the role of TEMHDFs in solidification process began shortly after Shercliff's study, because it had been already known ${ }^{[12]}$ that thermoelectric currents inherently exist at the solid-liquid interface. Initially in crystal growth $^{[13]}$ and secondly in solidification of metals, ${ }^{[14]}$ the existence of TEMHDFs has been confirmed, and studies on their influence have been undertaken. ${ }^{[15-18]}$

On the other hand, constrained by electric current continuity, thermoelectric currents should also flow through the solid phase. However, published research studies on the interaction between thermoelectric currents and static magnetic fields (which produces a kind of electromagnetic forces named TEMFs hereafter) in solid are scarce. Indeed, stresses in solids during solidification have a huge impact on the microstructural formation and then the performance of metallic materials. ${ }^{[19]}$ Although our previous studies show that the degeneration of columnar dendrites ${ }^{[20]}$ and the instability of solid-liquid interface ${ }^{[21]}$ in directional solidification under ultrahigh (up to 12T) magnetic fields may be attributed to TEMFs in solid phases, hitherto no definitive evidence for its existence could be found.

In this paper, we report live observations of the motion of solid grains during directional solidification of an Al-Cu alloy under a static magnetic field. Then, we analytically calculate the theoretical velocity of a spherical particle's motion driven only by TEMFs and compare this to the observed motions. The reasonable match reveals that TEMFs act on the solid grains and affect their motion trajectory. Corresponding 3D numerical simulations are carried out and validate our analytic calculation, which further confirms our conclusion that the motion of solid grains is the combined 
result of TEMFs and gravity. Hence, the results reported here can be regarded as the visible evidence for TEMFs and their influence during magnetic-assisted directional solidification.

\section{EXPERIMENTS}

A complete description of the sample, the experimental apparatus, and process has been detailed in Reference 22 . In brief, $\mathrm{Al}-4 \mathrm{wt} \% \mathrm{Cu}$ and $\mathrm{Al}-10 \mathrm{wt} \% \mathrm{Cu}$ alloys were used in this study. The dimensions of samples were about $200 \mu \mathrm{m}$ in thickness, $6 \mathrm{~mm}$ in width and $40 \mathrm{~mm}$ in length. The sample was sandwiched between two graphite foils and two molybdenum diaphragms fixed together with two clips, and this assembly was placed vertically at the center of a Bridgman furnace. The thermal gradient was imposed by two separate heating elements, and the directional solidification was controlled by applying the same cooling rate on these two heating elements. A permanent magnet mounted close to the Bridgman furnace provided a $0.08 \mathrm{~T}$ static magnetic field that was parallel to the beam and uniform within the sample.

In situ and real-time observations were carried out on beamline BM05 at the European Synchrotron Radiation Facility (ESRF), Grenoble. We took advantage of the high energy of X-ray beam $(17.5 \mathrm{keV})$ to obtain sufficient transmission and strong contrast between liquid and solid phases. The detector was the fast readout-low noise (FReLoN) CCD camera developed at the ESRF, which provided a good compromise between large fields of view $\left(15 \times 6 \mathrm{~mm}^{2}\right)$ and satisfactory spatial resolution (pixel size: $7.47 \times 7.46 \mu \mathrm{m}^{2}$ ). The high intensity of synchrotron radiation made it possible to record images with enough contrast in a short timescale ( $\sim 0.7$ second $)$. This was sufficient to study the motion of solid grains in directional solidification. Details of this synchrotron X-ray radiography setup can be found in Reference 23.

\section{RESULTS AND DISCUSSIONS}

We performed magnetic field-assisted directional solidification in the columnar regime for the first set of experiments, and found not only the dendritic growth but also the motion of fragments or nucleated grains. By superimposing several successive images captured during the directional solidification into one picture, the trajectory of grain motion is revealed as shown in Figure 1 (the white dotted line with arrows). These observations, respectively, correspond to the directional solidification conducted under positive (parallel to the beam) and negative (anti-parallel to the beam) $0.08 \mathrm{~T}$ static magnetic fields. We can find that the direction of grain motion is reversed when the magnetic field is applied in the opposite direction [see Figures 1(b) and (d)]. Since solid grains are generally subjected only to gravity and hydrodynamic forces (when flows in the melt exist), when no external field is applied, the observed grain motions indicate that those solid grains under a transverse magnetic field may be subjected to the other forces that orient along the $y$-axis, and their direction depends on the direction of the applied magnetic field. TEMF is a reasonable interpretation under the present conditions. Moreover, based on studies of the origin of liquid channels in solidification, ${ }^{[24,25]}$ the appearance of significant liquid channels in the bulk dendrites as shown in Figures 1(a) and 1(c) suggests the existence of flows in the melt. For the present case, these flows are TEMHDF ${ }^{[16,26]}$ because buoyancy-induced convection is negligible in such thin sample. ${ }^{[27]}$ The appearance of TEMHDF proves that interaction between thermoelectric currents and the applied magnetic field does take place in these magnetic field-assisted directional solidifications. Thus, it is reasonable to attribute those $y$-axis directional forces revealed by the observed motion of solid grains to TEMFs.

As we mentioned in the previous paragraph, solid grains are subject to hydrodynamic forces when TEMHDFs exist in the melt, therefore we performed a second set of directional solidifications with Al-10wt\% $\mathrm{Cu}$ alloy and lower thermal gradients to achieve sole equiaxed grain growth to minimize impact due to TEMHDFs. Superimposed pictures in Figure 2 show the trajectories (red arrows) of the equiaxed grain motions observed in real time during magnetic field-assisted directional solidifications conducted with different thermal gradients. The grains almost vertically sink downward when the thermal gradient is zero [see Figure 2(a)] because their density is higher than the surrounding melt for $\mathrm{Al}-10 \mathrm{wt} \% \mathrm{Cu}$ system. ${ }^{[28]}$ Increasing the thermal gradient to $500 \mathrm{~K} / \mathrm{m}$ makes the grains migrate toward the left [see Figure 2(b)], which suggests the appearance of negative $y$-axis-oriented TEMFs on the equiaxed grains because thermoelectric currents intrinsically exist at the solid-liquid interface in the presence of a thermal gradient. We can also find that the angle between the mean trajectories (yellow arrows) of equiaxed grain motions and the negative $y$-axis becomes smaller when the thermal gradient increases to $2000 \mathrm{~K} /$ $\mathrm{m}$ [see Figure 2(c)], which reveals that the magnitudes of TEMFs on the grains increase with the increasing thermal gradient. Moreover, the direction of equiaxed grain motions is reversed when a negative thermal gradient is imposed as shown in Figure 2(d). This demonstrates that the direction of TEMFs on the grains correlates with the direction of thermal gradient as well.

To further confirm that the observed grain motions do in fact result from TEMFs, we carried out an analytic calculation of the velocity of a spherical particle's motion driven only by TEMFs under steady-state conditions and compared this to the experimental results. The sample geometry used by in situ experiments is illustrated in Figure 3(a), and as shown in Figure 3(b), we simplify an equiaxed grain as a spherical particle to be the analytic model. Together with the physical property differences between liquid and solid phases, the thermal gradient by definition causes a temperature difference between the top and the bottom of the particle (with $T_{1}>T_{2}$ ), which gives rise to the appearance of thermoelectric currents [black dotted circle with arrow 


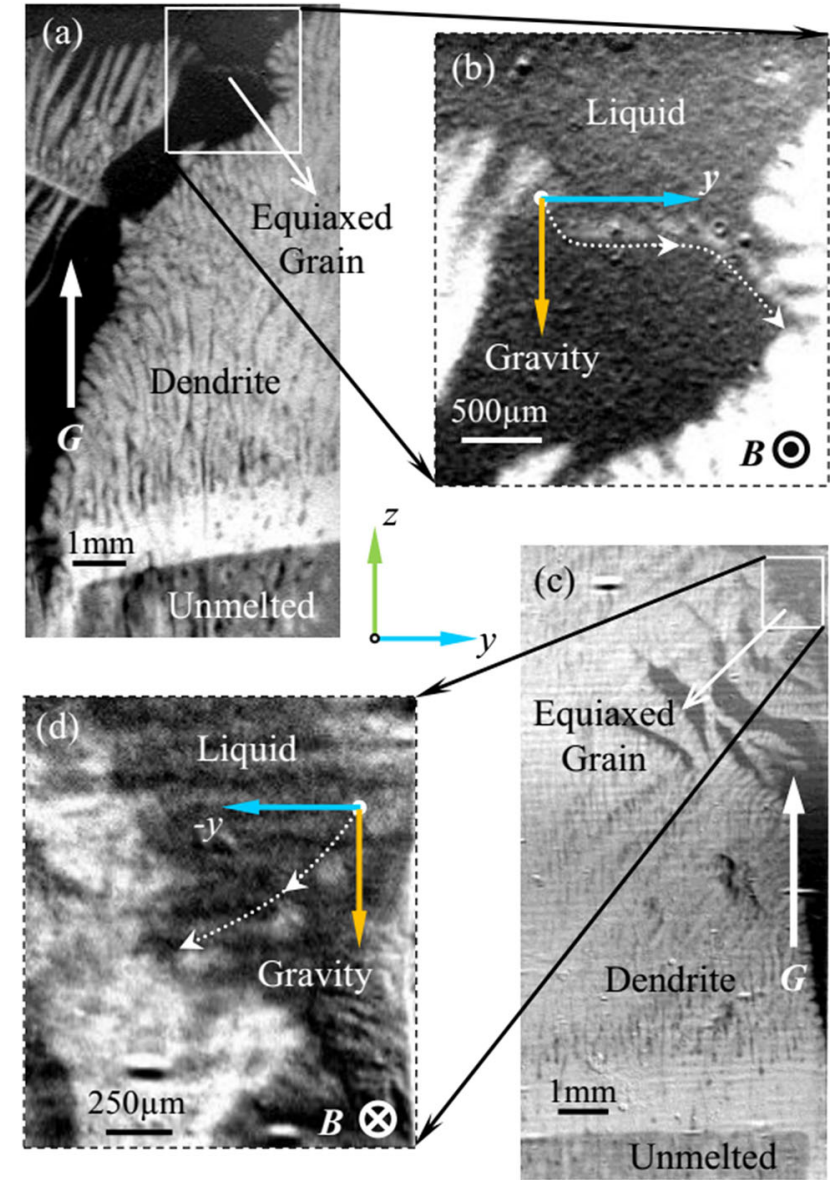

Fig. 1-Superimposed pictures of successive images captured during directionally solidifying $\mathrm{Al}-4 \mathrm{wt} \% \mathrm{Cu}$ alloys under positive $(a)$ and negative $(c)$ 0.08T static magnetic fields, $B .(b)$ and $(d)$ are the magnified views of the regions framed by the boxes, respectively, in (a) and (c). ( $G=3000 \mathrm{~K} / \mathrm{m}$; Cooling rates are $2 \mathrm{~K} / \mathrm{min})$.

in Figure 3(b)] in accordance with the Thomson-Seebeck effect. We first assume that the particle size is small enough to neglect the influence of the container wall, the thermal conductivities are identical in each phase, and the liquid around the particle is infinite. Therefore, the single $y$-component of the terminal velocity of the spherical particle driven only by the balance between TEMF and Stokes drag can be calculated according to Reference 22:

$$
U=\frac{4\left(S_{l}-S_{s}\right) \sigma_{s} \sigma_{l}}{9\left(2 \sigma_{l}+\sigma_{s}\right) \mu} \mathrm{GBR}^{2},
$$

where $U$ is the calculated velocity component along the $y$-axis $(\mathrm{m} / \mathrm{s}) ; \sigma$ and $S$, respectively, denote electric conductivity and absolute thermoelectric power; subscripts $s$ and $l$ indicate solid and liquid phases, respectively $\left(\sigma_{s}=4.0 \times 10^{6} \Omega / \mathrm{m}, \sigma_{1}=7.9 \times 10^{6} \Omega / \mathrm{m}, S_{s}=\right.$ $\left.-1.5 \times 10^{-6} \mathrm{~V} / \mathrm{K}, S_{l}=-2.25 \times 10^{-6} \mathrm{~V} / \mathrm{K}\right) ; \mu$ is the dynamic viscosity of the liquid $\left(2.9 \times 10^{-3} \mathrm{~Pa} \mathrm{~s}\right)$; $G$ is the thermal gradient $(\mathrm{K} / \mathrm{m}) ; B$ is magnetic field flux intensity (in $T$ ); and $R$ is radius of the spherical particle (in $\mathrm{m}$ ). The calculated velocities versus the radii of particles were plotted and are shown in Figure 3(c) for different thermal gradients and with an unchanged magnetic field. The $y$-axis directional velocities of the observed solid grain motions under the same conditions were estimated by dividing the motion trajectory length by the time interval between two images, and the measured velocities of detected grains were also plotted versus their radii in Figure 3(c) (the experimental determination of the grain radius can be found in Reference 22). We can note that our calculation and the observation results are in reasonable agreement. The discrepancy should be attributed to the fact that the real grains are not spherical, and additionally, the very thin crucible partially blocks the grain motion, particularly the one with large size. Thus, this agreement confirms that TEMFs do indeed act on the solid grains and explain both the amplitude and direction of the observed grain motions.

To validate the calculations and understand in more depth, the discrepancy between them and the experiments, we carried out 3D simulations of TEMFs using a
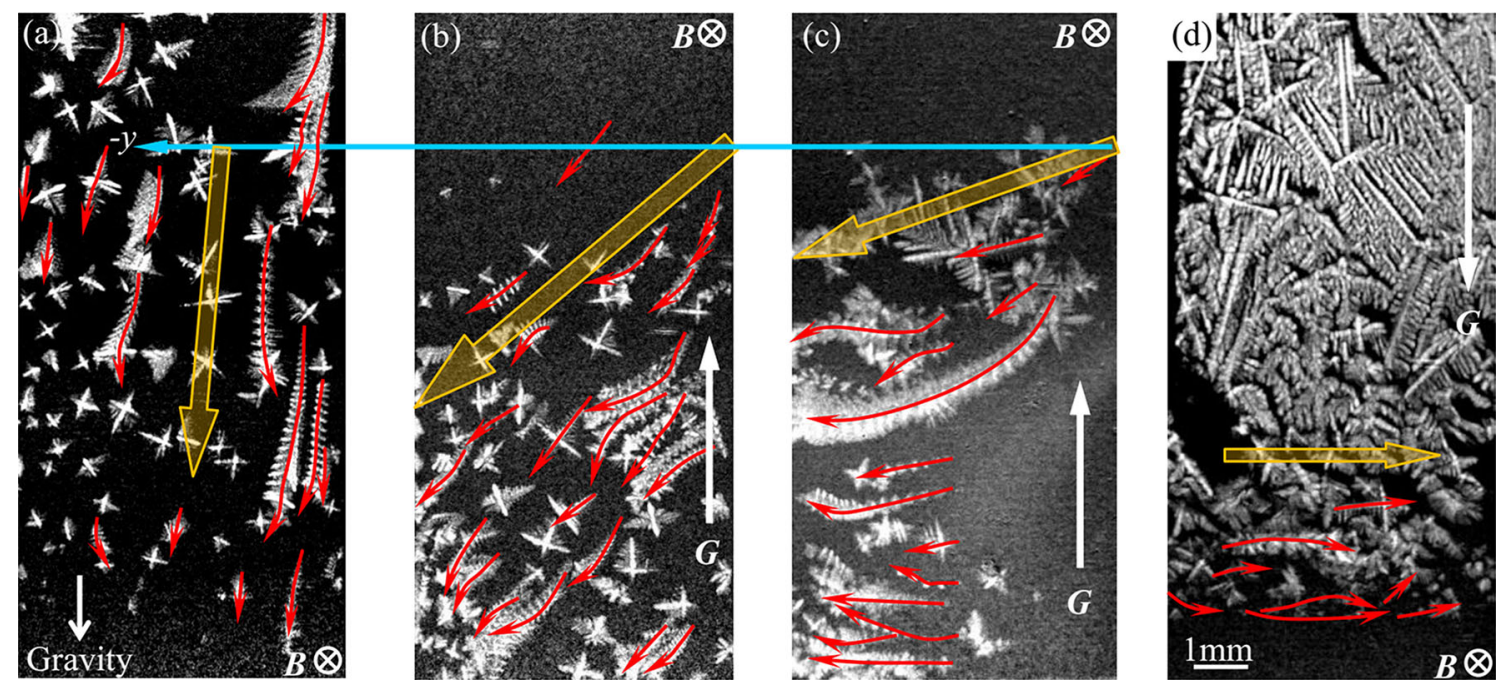

Fig. 2-Superimposed pictures showing equiaxed grain motion during directional solidifications of Al- $10 \mathrm{wt} \% \mathrm{Cu}$ alloys with different thermal gradients $(a) G=0 \mathrm{~K} / \mathrm{m},(b) G=500 \mathrm{~K} / \mathrm{m},(c) G=2000 \mathrm{~K} / \mathrm{m},(d) G=-2000 \mathrm{~K} / \mathrm{m} .(B=-0.08 \mathrm{~T}$; Cooling rates are $2 \mathrm{~K} / \mathrm{min})$. 


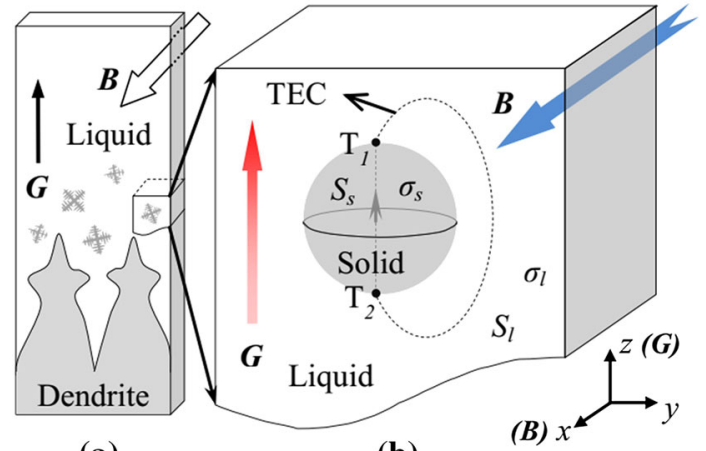

(a)

(b)

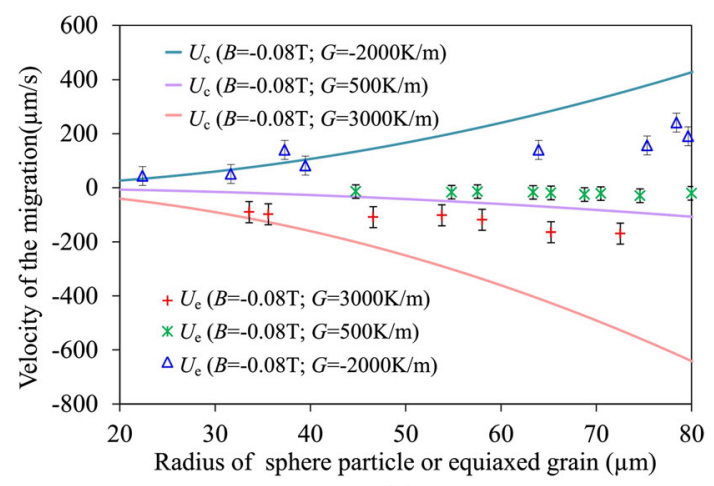

(c)

Fig. 3-(a) Sketch of sample geometry used by in situ experiments. Directional solidification proceeds with a positive (upward) thermal gradient and under a $0.08 \mathrm{~T}$ static magnetic field oriented along the positive $x$-axis; (b) Sketch of model geometry used in the calculating the velocity of the spherical particle driven only by TEMFs acting on it and illustration on how thermoelectric currents distribute; $(c)$ Comparison between the analytically calculated velocities and the measured velocities of the observed grains (Uc is the calculated velocity and $\mathrm{Ue}$ is the experimentally measured $\mathrm{y}$-component velocity).

finite-element method-based commercial code COMSOL Multiphysics (details of simulation method can be found in Reference 22). The computed TEMF distributions for, respectively, the spherical and equiaxedgrain-like particles are presented in Figures 4(a) and (b).

For the sphere, we can see that the TEMFs act on the solid in the positive $y$-axis direction, which agrees with the previous analytic solution, and furthermore, the total force obtained by making a volume integration of the computed TEMFs on the sphere, $F_{\text {total }}=1.32 \times$ $10^{-9} \mathrm{~N}$, is well consistent with the analytically calculated one of $F_{\text {total }}=1.02 \times 10^{-9} \mathrm{~N}$. This validates the analytic calculations. For the equiaxed grain, which have the same volume with the sphere, the computed TEMFs on it also orient toward positive $y$-axis. The total TEMF on an equiaxed-grain-like particle $F_{\text {total }}=$ $0.69 \times 10^{-9} \mathrm{~N}$ is smaller than that on a spherical particle, $F_{\text {total }}=1.32 \times 10^{-9} \mathrm{~N}$. This contributes to the discrepancy appearing in Figure 3(c) and explains why the real motion velocity is slower than the calculated one. Besides, we must notice that TEMFs also act in the liquid phase and may generate flows (TEMHDFs) around the particle, and therefore, their flow fields were also computed. The pressure fields caused by

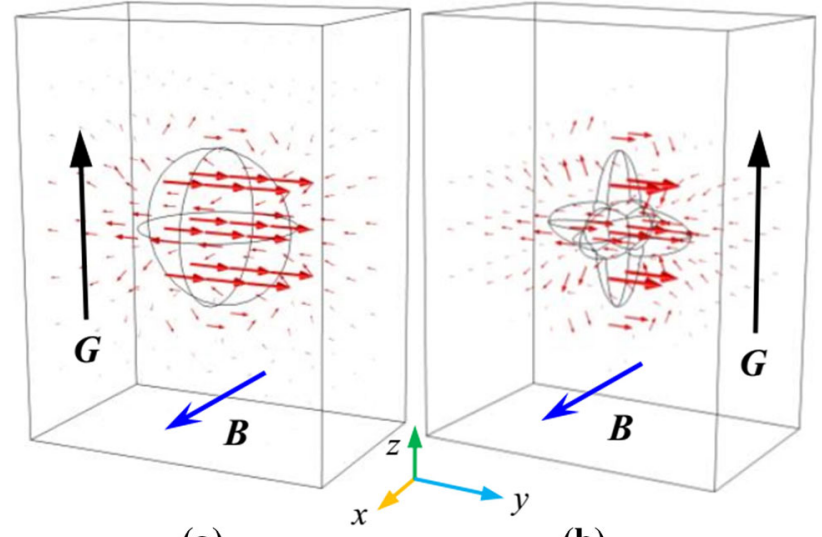

(a)

(b)

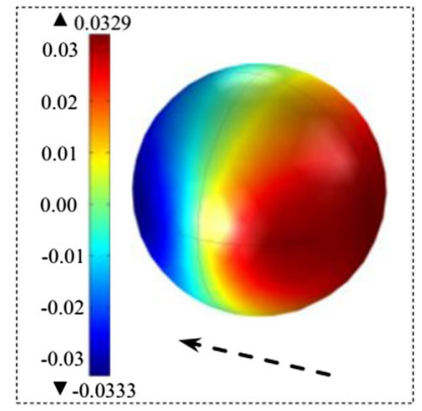

(c)

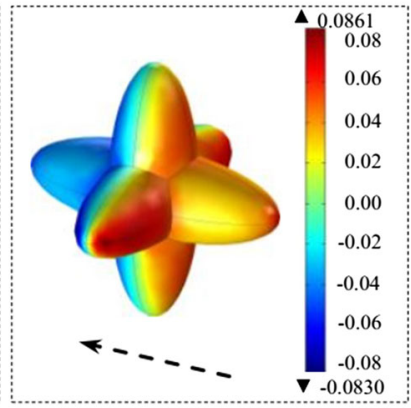

(d)
Fig. 4-Computed TEMF distributions in both liquid and solid for the spherical particle with the diameter of $150 \mu \mathrm{m}(a)$ and the equiaxed grain $(b)$ cases; and pressure field (in $\mathrm{Pa}$ ) on the spherical particle $(c)$ and the equiaxed grain $(d)$. These pressures result from flows in the melt around the solid particles, and those flows are caused by TEMFs in liquid. Thermal gradient, $\mathrm{G}$, is $3000 \mathrm{~K} / \mathrm{m}$ and the applied magnetic field, $B$, is $0.08 \mathrm{~T}$ in the positive $x$-axis direction.

TEMHDFs on the particles are shown in Figures 4(c) and (d). As indicated by the black dotted arrows, these pressures tend to counterbalance the TEMFs on the particle, i.e., in the opposite direction to that of the TEMFs (to the left in the present case). This may also contribute to the discrepancy in Figure 3(c) because the analytic calculations did not consider the influence of TEMHDFs around the particle.

\section{CONCLUSIONS}

In summary, this paper provides in situ and real-time observations of the motion of solid grains during magnetic field-assisted directional solidification of $\mathrm{Al}-\mathrm{Cu}$ alloys, which is the first visible evidence for the existence of TEMFs in solid and its impact. The dependencies on applied thermal gradient and magnetic field expected for the TEMFs in solid grains are clearly observed. Good agreement between the analytic solutions and observations reveals that TEMFs act on the solid grain and affect its motion trajectory. 3D numerical simulations of TEMFs as well as resulting TEMHDFs validate the analytic calculations, which further confirm our conclusion that the observed motion 
of solid grains is the combined result of TEMFs and gravity.

\section{ACKNOWLEDGMENTS}

We thank T. Lafford, E. Fene, and X. Guichard (ESRF, BM05) for their help during the experiment, and D. S. Eastwood (The University of Manchester, UK) for his help on editing the English language. This study was partly supported by the National Natural Science Foundation of China (Nos. 51604171 and 51690162), the Science and Technology Commission of Shanghai Municipality (No. 17JC1400602), and the fund of the State Key Laboratory of Solidification Processing in NWPU (Nos. SKLSP201706 and SKLSP201602).

\section{REFERENCES}

1. W.V. Youdelis, D.R. Colton, and J. Cahoon: Can. J. Phys., 1964, vol. 42, pp. $2238-58$

2. A. Goetz: Phys. Rev., 1930, vol. 35, pp. 193-207.

3. Z. Sun, M. Guo, J. Vleugels, O. Van der Biest, and B. Blanpain: Curr. Opin. Solid State Mater. Sci., 2012, vol. 16, pp. 254-67.

4. X. Li, Y. Fautrelle, and Z.M. Ren: Scripta Mater., 2008, vol. 59, pp. $407-10$

5. P. Deng and J.G. Li: Scr. Mater., 2006, vol. 55, pp. 747-50.

6. S. Karagadde, L. Yuan, N. Shevchenko, S. Eckert, and P.D. Lee: Acta Mater., 2014, vol. 79, pp. 168-80.

7. Q. Wang, T. Liu, A. Gao, C. Zhang, C.J. Wng, and J.C. He: Scr. Mater., 2007, vol. 56, pp. 1087-90.

8. H.P. Utech and M.C. Flemings: J. Appl. Phys., 1966, vol. 37, pp. 2021-24.
9. H.A. Chedzey and D.T.J. Hurle: Nature, 1966, vol. 210, pp. $933-$ 34.

10. J.A. Shercliff: J. Fluid Mech., 1979, vol. 91, pp. 231-51.

11. M.A. Jaworski, T.K. Gray, M. Antonelli, J.J. Kim, C.Y. Lau, M.B. Lee, M.J. Neumann, W. Xu, and D.N. Ruzic: Phys. Rev. Lett., 2010, vol. 104, p. 094503(1-4).

12. A.E. Mikelson and Y.K. Karklin: J. Crystal Growth, 1981, vol. 52, pp. 524-29.

13. L.A. Gorbunov: Magnitnaya Gidrodinamika, 1987, vol. 4, pp. 6575.

14. T. Alboussière, R. Moreau, and D. Camel: C. R. Acad. Sci. Paris, 1991, vol. 313, pp. 749-55.

15. P. Dold, F.R. Szofran, and K.W. Benz: J. Crystal Growth, 2006, vol. 291, pp. 1-7.

16. P. Lehmann, R. Moreau, D. Camel, and R. Bolcato: Acta Mater., 1998, vol. 46, pp. 4067-79.

17. X. Li, A. Gagnoud, Z.M. Ren, Y. Fautrelle, and R. Moreau: Acta Mater., 2009, vol. 57, pp. 2180-97.

18. A. Kao: Metall. Mater. Trans. A, 2015, vol. 46, pp. 4215-33.

19. I. Durand, K. Kassner, C.Q. Mishbah, and H. Muller-krumbhaar: Phys. Rev. Lett., 1996, vol. 76, pp. 3013-16.

20. X. Li, Z.M. Ren, Y. Fautrelle, A. Gagnoud, Y.D. Zhang, and C. Esling: Scr. Mater., 2009, vol. 60, pp. 443-46.

21. X. Li, Y.D. Zhang, Y. Fautrelle, Z.M. Ren, and C. Esling: Scr. Mater., 2009, vol. 60, pp. 489-92.

22. J. Wang: Ph. D thesis, University of Grenoble, Grenoble, France, 2013.

23. H. Nguyen-Thi, H. Jamgotchian, J. Gastaldi, J. Hartwig, T. Schenk, H. Klein, B. Billia, J. Baruchel, and Y. Dabo: J. Phys. D: Appl. Phys., 2003, vol. 36, pp. A83-A86.

24. M.G. Worster and J.S. Wettlaufer: J. Phys. Chem. B, 1997, vol. 101, pp. 6132-36.

25. L. Yuan and P.D. Lee: Acta Mater., 2012, vol. 60, pp. 4917-26.

26. J. Wang, Y. Fautrelle, Z.M. Ren, H. Nguyen-Thi, G. Salloum-Abou-Jaoude, G. Reinhart, N. Mangelinck-Noël, X. Li, and I. Kaldre: Appl. Phys. Lett., 2014, vol. 104, pp. 1-4.

27. N. Bergeon, D. Tourret, L. Chen, J.-M. Debierre, R. Guerin, A. Ramirez, B. Billia, A. Karma, and R. Trivedi: Phys. Rev. Lett., 2013, vol. 110, p. 226102 (1-4).

28. H. Nguyen-Thi, A. Bogno, G. Reinhart, B. Billia, R.H. Mathiesen, G. Zimmermann, Y. Houltz, K. Loth, D. Voss, A. Verga, and F. de Plascale: J. Phys.: Conf. Ser., 2011, vol. 327, pp. 1-11. 